\title{
Las remesas y el bienestar en las familias de migrantes
}

\author{
Miguel Ángel Corona*
}

\section{Resumen}

En este artículo se presentan los resultados de una investigación sobre el uso de las remesas en familias de migrantes y la repercusión en su bienestar, así como sobre la migración como estrategia para sobrevivir, adaptarse y participar en la globalización, dentro de un contexto económico nacional desfavorable. Nos situamos particularmente en la comunidad de San Jerónimo Coyula, en el estado de Puebla, caracterizada por su alta migración. Los resultados obtenidos muestran que las remesas en los hogares encuestados tuvieron un efecto positivo en su desarrollo y bienestar, por lo que se infiere que la migración ha sido y seguirá siendo la estrategia de los hogares para integrarse a los flujos de la globalización y alejarse de los riesgos del empobrecimiento en el lugar de origen.

\begin{abstract}
This article explores the use of remittances in migrant households of San Jeronimo Coyula, Puebla, and its impact on their well-being. San Jeronimo Coyula is the hometown of many migrants of the state of Puebla living in New York. Results show that households believe that remittances have significantly improved their life conditions. It is inferred that migration has been and will continue to be the strategy of households to join in globalization flows and move away from the risks of impoverishment in their place of origin.
\end{abstract}

Palabras clave: remesas, Coyula, Puebla, migración, bienestar y seguridad en el futuro. Key words: remittances, Coyula, Puebla, migration, life conditions, future prospects.

* Doctor en Administración de Organizaciones. Profesor de la Universidad Iberoamericana, Puebla. 


\section{Introducción}

L

as remesas son producto de la migración, fenómeno social que ha estado presente durante toda la historia de la humanidad. Por su naturaleza, el movimiento de personas de un lugar a otro es muy dinámico y tiene consecuencias en muchos ámbitos de la vida social, tanto para los lugares de origen como en los de destino, una característica común de este desplazamiento es la búsqueda de bienestar y mejores condiciones de vida.

En nuestro país el flujo migratorio hacia los Estados Unidos es el más importante, tanto por el número de personas como por el monto de remesas que genera. Además, este circuito migratorio está enmarcado por la historia de las relaciones bilaterales con el país vecino del norte. Cabe mencionar por un lado, que la economía estadounidense ha demandado la mano de obra mexicana para mantener su nivel de actividad económica y por el otro, que México ha tenido períodos de crisis económicas y lento crecimiento a partir de los años ochenta que han provocado desempleo y bajos salarios, situación que se ha vuelto la principal motivación para la migración a los Estados Unidos. Este es el caso de los circuitos migratorios relativamente recientes en el centro y sur del país, entre los que se encuentra el de la región de Atlixco en el Estado de Puebla.

Con este trabajo se plantea un acercamiento a la realidad de los hogares que reciben remesas como los de San Jerónimo Coyula, una localidad próxima a la ciudad de Atlixco en Puebla, que se ha convertido en el centro más relevante de la región. Para tener una aproximación de su importancia mencionaremos que en el 2003 Atlixco era uno de los municipios que más remesas recibía en la entidad, ${ }^{1}$ en el 2005 las remesas que recibieron las familias en Puebla totalizaron 1,133 millones de dólares, equivalentes al 4\% del producto interno bruto estatal de ese año, en el 2011 las remesas ascendieron a 1,465.1 millones de dólares, ${ }^{2}$ representando el $6.4 \%$ del total

1 Los municipios con más recepción de remesas eran Puebla, Acatlán, Izúcar de Matamoros, Tepeaca, San Martín Texmelucan, Atlixco, Chiautla de Tapia, San Andrés y San Pedro Cholula. Presidencia de la República. Consultado 24 Enero 2012. Disponible en: http:/ / fox.presidencia.gob.mx/buenasnoticias / ?contenido $=5112 \&$ pagina $=379$

2 Banco de México, 2012. Cuadro resumen Ingresos por remesas familiares y por entidad federativa. Consultado 28 marzo 2012. Disponible en: http:/ / www.banxico.org.mx/SieInternet/ consultarDirectorioInternetAction.do?accion $=$ consultarCuadroAnalitico\&idCuadro=CA79\&s $\underline{\text { ector }=1 \& \text { locale }=\mathrm{es}}$ 
nacional y como porcentaje del PIB estatal subió al 5\%. Con esto no hay duda que se trata de una de las regiones que más influencia recibe del exterior, tanto por el movimiento de la población, como por la recepción de remesas.

\section{La comunidad de San Jerónimo Coyula}

San Jerónimo Coyula es una localidad ubicada en las faldas del volcán Popocatépetl, en el Valle de Atlixco, Estado de Puebla, con algunos antecedentes migratorios del Programa Bracero, que a partir de los años ochenta se incorpora a los circuitos migratorios relativamente recientes a nivel nacional, ${ }^{3}$ y cuyo estudio representa un caso importante para entender los efectos de las remesas en el bienestar de los hogares.

Es importante destacar que la gran mayoría de los migrantes poblanos provienen de comunidades rurales. En el Estado de Puebla se reconocen zonas con tradición migratoria hacia el extranjero, en donde existen proporciones significativas de personas que actualmente están laborando en el área de Nueva York - Nueva Jersey, este es el caso de la región de la Mixteca, pero también han estado cobrando importancia otras en el norte y cerca de Puebla, capital del Estado, como la región de Atlixco (Cortina y Gendreau, 2004).

De acuerdo a una encuesta realizada por este autor en San Jerónimo Coyula en el 2006, la mayoría de las familias contaba por lo menos con un miembro en Nueva York, es decir, el 68 \% de los hogares tenían un familiar en Estados Unidos. ${ }^{4}$

\section{Relevancia del tema}

Desde la perspectiva de las familias y de los migrantes mexicanos, la respuesta a las grandes asimetrías entre las economías de los países y a los cambios estructurales originados por el proceso de apertura e integración

3 Existen al menos tres tipos de circuitos migratorios: el histórico, que se localiza en las regiones con migración más antigua como Zacatecas, Michoacán y Jalisco. El indígena, que se ubica en Oaxaca y Guerrero principalmente. El emergente, que contempla regiones en varios estados del centro y sureste del país, donde incluso se combina con los otros circuitos. La migración del estado de Puebla, con excepción de la Mixteca y la Sierra Norte, se considera como emergente.

4 Véase la sección de metodología líneas abajo. 
económica, han sido estrategias de sobrevivencia que ya no contemplan sólo el ámbito local y nacional (Ratha, 2003). La forma en que muchas de las familias mexicanas se han podido incorporar a los flujos económicos de la globalización ha sido gracias a la migración hacia los Estados Unidos. A través de la migración el dinero de los grandes centros económicos del norte puede bajar al sur, mediante la parte de los salarios que se envían como remesas (Corona, 2008). De esta manera es posible que los hogares que las reciben aumenten sus niveles de ingreso, se alejen del riesgo del empobrecimiento y se potencien para mejorar su bienestar.

Visto desde este ángulo, el bienestar se relaciona con la mejoría de las condiciones de vida de la familia, considerando en primer lugar la alimentación y la vestimenta, en segundo lugar la salud y la educación, seguidas de la vivienda. Bajo esta circunstancia se concibe un bienestar material que se logra con la obtención de mayores ingresos. Así la migración se convierte en la posibilidad de obtener un ingreso mayor en el lugar de destino, que permite remitir una parte al lugar de origen para mejorar la situación de los que se quedan. Por lo tanto, en una primera instancia se esperaría que todos los hogares que reciben remesas mejoren su bienestar o al menos su expectativa de ello. Sin embargo, el proceso no es tan simple, el bienestar va más allá de lo material.

Para entender la trascendencia del fenómeno migratorio es necesario revisar primero las causas que lo originan y después el impacto que tienen las remesas sobre el bienestar de las familias, considerando la forma cómo las utilizan, sin dejar de intentar una aproximación al bienestar y su interpretación.

\section{Migración y remesas}

Existen varios planteamientos teóricos para explicar por qué las personas emigran. La teoría neoclásica refiere como la causa principal la existencia de lugares que atraen mano de obra por sus necesidades de funcionamiento y crecimiento, al tiempo que otros lugares la expulsan debido a su incapacidad para generar empleos bien remunerados y expectativas de progreso para sus habitantes. El principal indicador de estas asimetrías es el diferencial salarial entre los dos lugares, regiones o países. Los supuestos en que se basa están relacionados con la decisión individual y un balance entre los costos y los beneficios por migrar (Durand y Massey, 2003). La nueva teoría de la migración laboral, parte del supuesto que la decisión 
se hace en el núcleo familiar, considerando su estructura y potencialidad en función de sus miembros. Responde también a un sentimiento de pobreza relativa frente a otros grupos familiares de la comunidad y supone un balance entre costos y beneficios (Taylor, 1999). En este planteamiento la noción de bienestar se puede deducir como contraparte de una insatisfacción del grupo dentro de la comunidad, al ver a otras familias con migrantes que progresan al recibir remesas de los miembros de su grupo que trabajan en el extranjero.

Se ha encontrado que el diferencial salarial entre dos países es una buena explicación de la migración (Borjas, 2000), ya que el individuo va en busca de recursos que no consigue en su lugar de origen para sobrevivir en un primer momento, pero también están los que afirman que las personas migran para mejorar su situación patrimonial y de carencia relativa con relación a otras familias en su comunidad (Stark y Taylor, 1991). A partir de los motivos por los cuales se emigra, es posible derivar la necesidad de generar excedentes de un salario internacional para remitirlos al lugar de origen donde se encuentra la familia (Taylor, 1999). En los estudios realizados los principales motivos que se han encontrado son: por altruismo, para formar un patrimonio y para ayudar a las obras de la comunidad. En todos los casos se refiere a la carencia de recursos económicos que financien los gastos corrientes de los hogares, las necesidades de seguridad para el futuro y la formación de capital social comunitario.

Los estudios sobre las remesas se han enfocado principalmente en dos sentidos: a observar y analizar los efectos en los países de origen en función de ser un elemento que contribuya a disminuir las desigualdades y por lo tanto convertirse en un medio para el desarrollo (Taylor, 1992; Lozano, 2004) y en segundo lugar a analizar las remesas como parte de un salario internacional (Canales, 2008), que representa un medio de sobrevivencia para los hogares que las reciben alejándolas del riesgo del empobrecimiento. Complementariamente existe otro enfoque, menos utilizado, que considerando el espacio trasnacional identifica otros factores de carácter estructural en el lugar de destino que combinados con características personales y sociales condicionan la temporalidad de la estancia, el monto, frecuencia y destino de los envíos de remesas y con ello sus impactos. (Orozco, 2004; Corona, 2008). En síntesis estos enfoques tratan de explicar una realidad que tiene muchas facetas, pero que interpela por los impactos que tiene al nivel de las comunidades, pero sobretodo de las familias en el lugar de origen.

Respecto a los impactos directos de las remesas sobre los hogares, los estudios se han concentrado en analizar el uso de las remesas, en ellos se ha determinado que no menos del $80 \%$ de las remesas recibidas se destina 
al consumo de bienes, en tanto que proporciones menores se destinan a construcción, salud, educación y muy poco para iniciar una fuente de ingreso (Canales, 2004; Corona, 2007). Lo cual resulta coherente en la lógica de un asalariado y el destino de su salario, con la característica de que en este caso el salario se obtuvo en otro país.

Por lo que se refiere al impacto en la comunidad y región, el dinero recibido vía remesas no se gasta mayoritariamente en la comunidad de origen, debido a que no cuenta con la suficiente oferta de bienes y servicios, por lo cual el gasto mayor se hace en los centros urbanos con mayor dotación de infraestructura y población que pueden soportar una oferta de bienes y servicios más diversificada (Corona, 2007), de esta forma los efectos benéficos del gasto son muy limitados para las comunidades de origen.

Todos estos elementos influirán sobre el bienestar de los individuos y de las familias, pero ¿en qué y cómo?

\section{El bienestar y su interpretación}

Bajo la idea de que el desarrollo es un proceso de mejoramiento en los niveles y la calidad de vida de la población, que por ende involucra aspectos de toda índole pero que hacen sentir bien a las personas, el bienestar es una consecuencia de ese proceso.

Sin embargo, su conceptualización no es fácil debido a que sus implicaciones van desde lo tangible a lo intangible, desde lo evidente hasta lo no evidente, desde lo material hasta lo espiritual, desde lo objetivo hasta lo percibido subjetivamente. El bienestar está relacionado con las condiciones que rodean a las personas, su cultura, sentimientos, percepciones y con los medios para gobernar su vida. En esta perspectiva multidisciplinaria Abraham Maslow (1943) hizo una buena aproximación de lo que comprende el bienestar, aportando su pirámide de las necesidades humanas, a partir de la cual es posible identificar sus tipos y jerarquías que van desde las fisiológicas hasta las de auto-realización, que involucran las estrictamente materiales hasta las muy subjetivas de realización personal, y en cuya satisfacción se ve reflejado el bienestar de las personas. Esta es una conceptualización que permite entender la multi-dimensionalidad de sentirse bien, desde una perspectiva compleja que comprende aspectos tanto objetivos como subjetivos.

En el mismo campo de la psicología, el bienestar se ha estudiado identificando los factores determinantes que involucra el individuo para considerar su vida de forma positiva, en otras palabras, el grado de satisfacción 
que tiene la persona sobre su vida (Seligman, 1999). El inconveniente es la subjetividad que envuelve y la dificultad de cuantificar y medir. A lo anterior hay que agregar que la sensación de bienestar también está influida por un elemento dinámico que supone una mejoría futura y que incluso se ha estudiado como forma de mantener la cohesión social en procesos de crecimiento o transición. Esto nos lleva a plantear que es necesario obtener elementos objetivos y subjetivos para poder aproximarnos a la noción de bienestar, que las personas sujetos del bienestar son capaces de opinar sobre su subjetividad (Ferrer-i-Carbonell, 2002b), y sus declaraciones, como reflejo de sus sentimientos, pueden ser incluso más poderosas que las propias mediciones objetivas.

Aunque se reconoce que el bienestar es un producto multidimensional, sus motores de empuje al menos en una primera etapa son el ingreso y el empleo, de acuerdo con algunos estudios son de las variables objetivas más relevantes ${ }^{5}$ (Ferrer-i-Carbonell, 2002a; Ravallion y Lokshin, 2000). Es necesario considerar también que el concepto de bienestar involucra un proceso de comparación con respecto a otros en cuanto a nivel económico y social por mencionar los principales, pero además es una comparación inter-temporal entre un estado previo y el actual del propio sujeto de estudio, es sin duda algo multidimensional, real o esperado, en el que el ingreso es una variable importante aunque no es la única.

Visto así, el estudio de las percepciones de los individuos sobre la noción de bienestar, a partir de los beneficios que han brindado las remesas, resulta importante para comprender la relación entre remesas y desarrollo, porque las decisiones de los hogares estarán motivadas por su percepción incluso si ésta no corresponde a la realidad. Es decir, a veces la percepción puede ser más optimista o pesimista de lo que es la realidad objetiva, todo ello debido a un criterio subjetivo de bienestar. En esta línea de percepciones se incluye el impacto que han tenido estos recursos sobre la alimentación, educación, salud y vivienda, desde la óptica y sentimiento de quienes reciben las remesas.

5 Ferrer-i-Carbonell (2002a) concluye que el ingreso del grupo de referencia es tan importante como el ingreso personal para la felicidad individual. Los individuos son más felices si su ingreso es mayor en comparación con el ingreso de su grupo de referencia. Mientras que Ravallion y Lokshin (2000b) encuentran una correlación entre bienestar económico subjetivo e ingreso de 0.11 para 1994, y de 0.20 en 1996. 


\section{Metodología}

La muestra de hogares encuestados resultó como un subproducto de un grupo de hogares seleccionados de manera aleatoria para elaborar la matriz de contabilidad social en la comunidad de San Jerónimo Coyula, junta auxiliar del municipio de Atlixco en Puebla, entre los meses de febrero y mayo de 2006. El número de hogares encuestados correspondió sólo a los que recibían remesas, que en total sumaron $45 .{ }^{6}$ Los tamaños del hogar se distribuyeron de la siguiente manera: el $65 \%$ de los hogares tenía de 4 a 8 miembros, el 33\% de 9 a 12 miembros, y el restante $2 \%$ de 13 a 16 miembros. El tamaño promedio de los hogares fue de 7.6 miembros.

Otra de las características importantes que puede influir en el bienestar de los hogares es la antigüedad de la migración, para cuando se levantó la encuesta el 22\% de los hogares declaró que el (o los) miembro(s) de su familia tenían en los Estados Unidos una antigüedad de 1 a 4 años, mientras que la gran mayoría (53\%) llevaba de 5 a 9 años de migración. Los restantes, $20 \%$ y $5 \%$ de los encuestados declararon que su familiar llevaba en los Estados Unidos de 10 a 14 años, y de 15 a 20 años respectivamente. Lo anterior es relevante porque la gran mayoría de los migrantes $78 \%$ tenían más de 5 años radicando en el país del norte, lo cual puede significar varias cosas, el desarrollo de la red social que proporciona tranquilidad de la estancia, certidumbre laboral, mejores ingresos, mayor capacidad de ahorro, regularidad en el envío de remesas y visión mediata de los planes a futuro, entre otras.

Es importante destacar que hace una década era más fácil venir de visita a la comunidad de origen y regresar a los Estados Unidos. Con el endurecimiento de la política migratoria de aquel país, el circuito de ida y vuelta de muchos coyuleños ha disminuido en su frecuencia aunque no así el envío de remesas.

Otra variable importante fue el estado civil del migrante, que se clasificó en dos grupos: los unidos, en donde se incluyeron a los casados y en unión libre, mientras que en los no unidos se agruparon a los solteros, separados, divorciados y viudos. Bajo esta clasificación el 82\% de los encuestados estaban unidos, lo que implicaba que tenían al menos un dependiente económico, el restante $18 \%$ no lo estaban, pero esto no significaba

6 Se sabe en la comunidad que gran parte de los coyuleños han emigrado hacia el Estado de Nueva York y que el asentamiento más prominente de ellos se ubica en Long Island, muy cerca de la ciudad de Nueva York. 
que no enviaran remesas a sus familiares, varios eran hijos que mandaban dinero a sus padres.

\section{El presupuesto familiar y las remesas}

Antes de pasar al análisis del uso de las remesas y su impacto en la percepción del bienestar de los hogares que las recibían, es muy importante presentar la estructura del ingreso y del gasto para identificar las fuentes de recursos y las asignaciones que hacían para sobrevivir y desarrollarse. Con respecto a los primeros, la principal fuente de ingreso eran las remesas por el trabajo de familiares en los Estados Unidos, que en promedio representaban el 73\% del total, seguía muy por abajo con el 7\% el trabajo como peón agrícola en la comunidad, luego los préstamos familiares con el 5\%, enseguida con el $4 \%$ respectivamente ingreso por trabajo realizado en la región y ayudas para la salud y prestaciones, continuaba con el 3\% el ingreso por trabajo no agropecuario, y con el 1\% respectivamente, los hogares obtenían ingresos por sus ahorros, por los programas de gobierno "Oportunidades" y "Progresa", por préstamos bancarios y por otros conceptos, tal como se muestra en la gráfica 1

\section{Gráfica 1. Estructura del ingreso de los hogares con remesas}

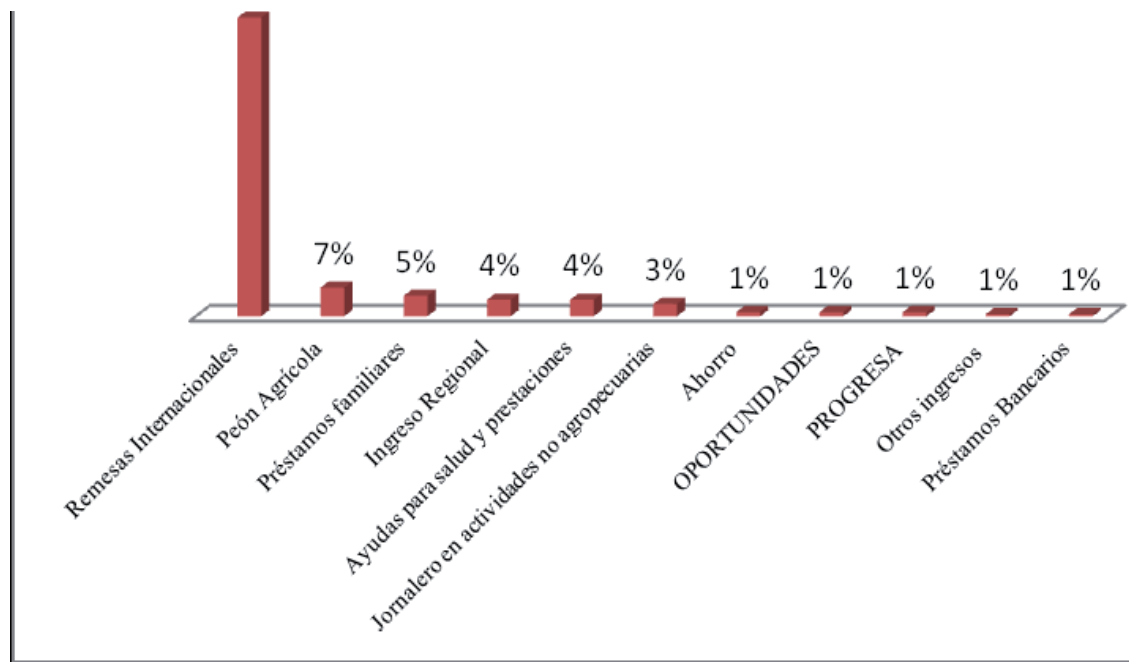

Fuente: Elaboración propia con base en cuestionarios para la MCS, San Jerónimo Coyula, 2006. 
En cuanto a la composición de sus gastos, el conjunto de hogares que recibía remesas asignaba en promedio el $45 \%$ a la alimentación, el $11 \%$ al pago de servicios de electricidad y telefonía, el 9\% a gastos de educación, el 7\%, a comprar bienes raíces y a cubrir gastos fuertes de hospitalización y medicamentos, el $4 \%$, a comprar combustibles y a pagar el viaje de los migrantes, el 3\% destinado a contribuciones para las fiestas religiosas y familiares, el $2 \%$ correspondían a erogaciones por tenencia, predial y otros impuestos, y finalmente el $1 \%$ iba directamente al pago del agua, salarios, compra de materiales de construcción y pago de consultas médicas, respectivamente. Ver gráfica 2.

\section{Gráfica 2. Estructura del egreso de los hogares con remesas}

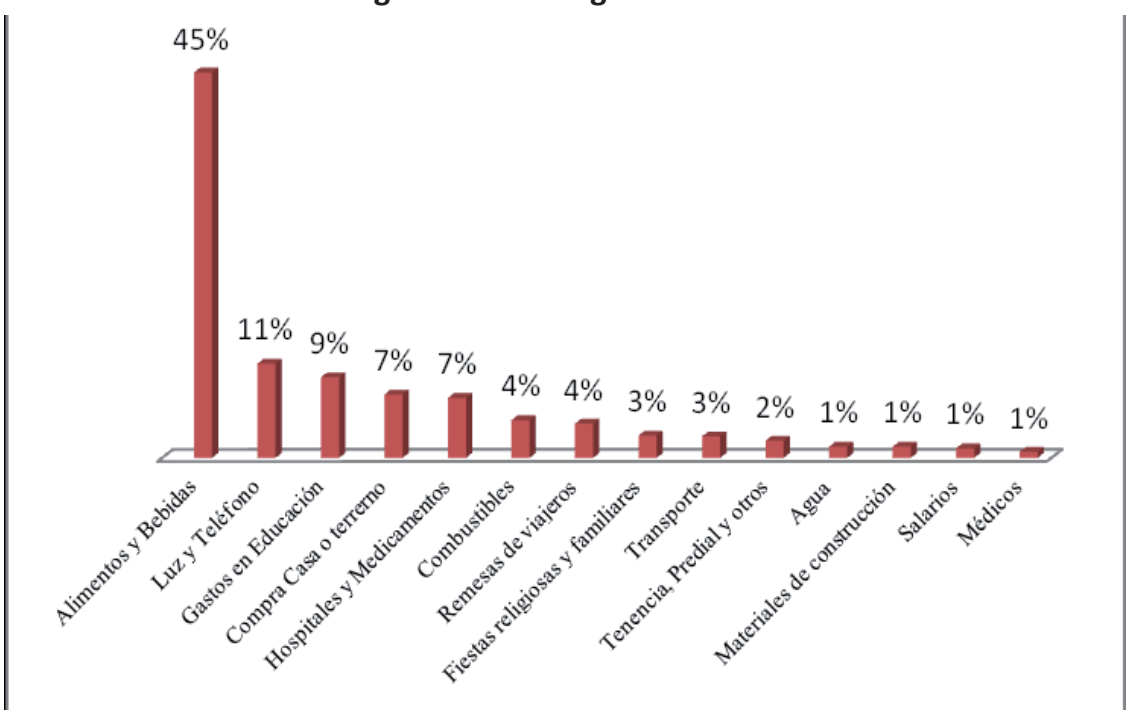

Fuente: Elaboración propia con base en cuestionarios para la MCS, San Jerónimo Coyula, 2006

Una vez hecho el análisis anterior es evidente el peso que tenían las remesas en los ingresos de los hogares que las recibían en San Jerónimo Coyula, por lo que era indispensable detallar sus usos, destinos e impacto en la percepción del bienestar.

Monto de las remesas

El primer dato relacionado con las remesas de los hogares fue el monto, en promedio ascendían a $\$ 29,091$ pesos anualmente. Con esta medición además fue posible estratificar la variable en bajos y altos ingresos por reme- 
sas, para efecto de análisis con tablas cruzadas. La forma en que recibían las familias sus remesas era significativa por la confianza que daba a los remitentes el mandar dinero al lugar de origen. En el 63\% de los hogares era mediante money orders (órdenes de pago), en el $24 \%$ utilizaban transferencias bancarias, lo cual implicaba que los migrantes y sus familias ya eran usuarios de servicios financieros. El restante 13\%, todavía recibía su dinero por medio de una persona que lo llevaba hasta la comunidad de origen, forma que poco a poco se ha ido dejando de usar debido al riesgo que representa y a la baja sustancial del costo de la comisión por el servicio de transferencia (Orozco, 2004) .

En la administración de las remesas es fundamental conocer si el migrante que envía estos recursos participa en la toma de decisión acerca del destino de los mismos. Los encuestados afirmaron que en el $87 \%$ de los casos sólo el migrante decide el destino de las remesas que envía. En el 13\% restante no interviene en esta decisión. Esto es importante porque la noción de bienestar de la familia se ve influida por la nueva visión del migrante de lo que debiera ser el bienestar en el lugar de origen.

\section{Uso y destino de las remesas}

En este punto es muy importante recordar que sólo se reporta el destino de las remesas, más no el del total del ingreso del hogar, por lo que se puede esperar que si el hogar utiliza la mayor parte de las remesas en productos básicos, estará subsanando una necesidad de sobrevivencia. Y conforme consuma productos no básicos estará alejándose de ese nivel. Ahora bien, la mejoría en el bienestar puede ser percibida desde las primeras etapas al aumentar su capacidad de consumo. A continuación se presenta información sobre la asignación de las remesas en rubros como alimentos, ropa y calzado, transporte, salud, educación, construcción y compra de tierras.

El principal rubro en que los hogares se gastaban las remesas era en alimentos, el $47 \%$ de los encuestados gastaba entre el 25 y $50 \%$ de sus remesas para comprar comida. Asimismo, el 40\% de los encuestados destinaban entre el 51 a 75\% del dinero que recibían en remesas para su alimentación. En una situación más extrema estaba el 9\% de los encuestados porque gastaban casi la totalidad de sus remesas (76 a 100\%) sólo para alimentación. En el caso opuesto estaba el $4 \%$ de los encuestados porque destinaban menos del 25\% de sus remesas al rubro de alimentos. Estos datos revelan que más de la mitad de los hogares encuestados destinaban proporciones muy considerables de las remesas en alimentos. 
El gasto en ropa y calzado fue el segundo en importancia porque forma parte de las necesidades básicas en un hogar. En el sondeo la mayoría de los encuestados (69\%) gastaba de 6 a $15 \%$ de las remesas en este rubro. El $20 \%$ destinaba entre 16 a $20 \%$ de remesas a ropa y calzado. El $11 \%$ de los encuestados destinaban menos del $5 \%$ a ropa y calzado. Ningún hogar reportó gastar más del $20 \%$ en este rubro. Debe recordarse que en muchas ocasiones los migrantes mandaban remesas en especie, que en la mayoría de los casos eran ropa y calzado junto con electrodomésticos u otros aparatos electrónicos.

En la asignación de las remesas a transporte no se reportó un gasto mayor, el 31\% de los encuestados no asignaban dinero a este concepto, el $67 \%$ destinaba hasta $10 \%$ de sus remesas, sólo el $2 \%$ gastaba de 11 a $20 \%$ de sus remesas en este rubro. Es necesario subrayar que en muchos casos las personas de San Jerónimo Coyula, se trasladaban a pie a lugares cercanos dentro de la misma comunidad.

En seguida se presenta el gasto en salud y educación. En realidad, el dinero asignado para este fin se puede considerar una inversión en capital humano, que es fundamental para que cada miembro del hogar pueda desarrollar todo su potencial productivo. El gasto en salud era muy importante en algunos de los hogares que recibían remesas. Incluso hubo casos en que la decisión de migrar estuvo influenciada por el estado de salud de algún familiar. En la encuesta, el $84 \%$ afirmó no destinar remesas al rubro de salud. Por su parte, el $6 \%$ asignaba el $20 \%$, el restante $7 \%$ gastó el $30 \%$ y el $3 \%$ asignaba $40 \%$ de las remesas a esta necesidad.

Como se mencionó anteriormente, un gasto relacionado con el desarrollo fue el de educación. En la encuesta, el 69\% contestó que no destinaba remesas a la educación, por su parte el 31\% restante si le asignaba de 5 a $10 \%$. En forma desglosada, el $11 \%$ asignaba $5 \%$ y el $20 \%$ destinaba $10 \%$.

Existe otro tipo de gastos que también representan una inversión en el estricto sentido de la palabra. El de construcción y en la compra de tierras son indudablemente las formas comunes en que las remesas se invierten después de que las necesidades básicas han sido satisfechas. Antes de pensar en utilizar las remesas para iniciar un negocio, las familias amplían su casa o adquieren bienes raíces. En el caso del gasto en construcción, el 58\% no asignaba recursos a este rubro, esto se debía probablemente a que eran hogares que todavía no tenían asegurado su consumo para sobrevivir y otros pocos ya habían pasado por esa etapa de construcción. El 16\% de los encuestados afirmó que asignaba de 10 a 29\% de las remesas para construir su casa. Un dato interesante fue que el $22 \%$ asignaba de 30 a $49 \%$. Con un 
porcentaje todavía mayor estaba el $4 \%$ de los encuestados que asignaba el $50 \%$ para construir o ampliar su vivienda.

La compra de tierras implica que el hogar ya está en la etapa de formar y/o expandir su patrimonio, en otros términos, está acumulando riqueza y ya tiene cierto nivel de ingresos regulares. Esto puede explicar por qué $87 \%$ de los hogares encuestados no reportaron asignar remesas a este rubro. Además para este caso particular, las asignaciones iban del 40 al 60\% porque el costo de la tierra era elevado en comparación con otro tipo de bienes. En forma detallada, el 7\% asignó el $40 \%$ de las remesas para comprar un terreno, mientras que el $4 \%$ destinó 50\% y sólo un 2\% invirtió el $60 \%$ de las remesas en la compra de tierras. Se puede observar que los porcentajes para estos rubros son bastante significativos.

El ahorro es una herramienta importante que a largo plazo puede mejorar las condiciones económicas del hogar, o en el peor de los escenarios puede mantener el consumo en tiempos de crisis económica o frente a alguna emergencia. Sin embargo, el principal requisito para que pueda formarse es que exista un excedente del ingreso que no se consuma, lo cual implica que el consumo diario está garantizado. Con base en lo anterior se puede explicar que el $67 \%$ de los hogares encuestados no ahorraban, en tanto que el 33\% ahorraron cierto porcentaje de sus remesas. En específico, el 18\% de los hogares encuestados ahorraron el 10\% de las remesas que recibían, el $2 \%$ el $5 \%$, y otro $2 \%$ el 15\%. Por último el 11\% ahorró el 20\% de sus remesas.

\section{Impacto de las remesas en los hogares}

En esta parte se muestran los resultados a las preguntas que indagan sobre la percepción de los hogares respecto al impacto que habían tenido las remesas en aspectos importantes de su vida. Esta percepción en cada caso tuvo como referente la situación que existía antes de que recibieran las remesas. Ver gráfica 3.

En principio se preguntó si las remesas habían mejorado, mantenido igual o empeorado su situación económica. Como podría esperarse, ningún hogar consideró que las remesas hubieran empeorado su situación. Por el contrario, un avasallador $71 \%$ de los hogares encuestados consideraron que habían mejorado su situación económica. El 29\% restante fue un poco más modesto y conservador porque declaró que las remesas habían mantenido igual su situación económica. Cabe recordar que en general, las personas que migran son las que experimentan carencias relativas en una 
comunidad y que las remesas que reciben les ayudan a compensar esas carencias paulatinamente.

En el tema de la alimentación, la percepción que tenían los hogares fue similar a la anterior, en cuanto a que ninguno declaró que el efecto de las remesas en este aspecto haya sido negativo. El 62\% mencionó que habían mejorado su alimentación, porque ya podían comprar más alimentos en especial carne, leche y huevos que tenían precios elevados en comparación con las frutas, verduras y legumbres. Sin embargo el restante $38 \%$ consideró que su alimentación no había cambiado con las remesas. En este punto puede haber varias explicaciones: la primera, que algunos de estos hogares mantenían su dieta tradicional, en un trabajo reciente comparando los patrones de consumo de alimentos de hogares que recibían remesas con los que no recibían, no se observaron diferencias significativas (Corona, 2009), segundo, que la remesa la ocupaban principalmente para comprar otro tipo de bienes, teniendo satisfecho su nivel de alimentación, tercero, que la remesa la ocupaban por encargo para comprar otro tipo de bienes, incluso sin haber considerado su nivel de alimentación.

En términos de salud, no hubo respuestas en las que haya empeorado la situación de salud. El 82\% de los encuestados coincidieron en que las remesas habían mejorado sus condiciones de salud. Mientras que el 18\% restante tenía una percepción más mesurada al declarar que su situación permanecía igual que antes de que su familiar migrara. Es importante resaltar en este rubro que algunas familias mencionaron que la ida de alguno de sus miembros hacia los Estados Unidos, fue motivada por la búsqueda de recursos para hacer frente a alguna enfermedad de origen congénito, crónico o de edad avanzada.

Respecto a la educación existía una percepción variada y polémica. En este aspecto hubo hogares (7\%) que consideraron que las remesas habían empeorado la situación de educación en su hogar. Su declaración estaba basada en el hecho de que las remesas habían provocado que los hijos no quisieran estudiar la preparatoria, porque desde que terminaban la secundaria, etapa con la que culmina la educación básica y obligatoria en México, querían irse a trabajar al país del norte. Esto estaba relacionado con la creencia de que migrar les permitiría hacer fortuna, situación estimulada en varios casos por la existencia de un familiar (padre, madre, hermanos, tíos y primos), que podía ayudarlos a encontrar trabajo en los Estados Unidos. Cabe recordar que al existir una red social se reduce el riesgo e incertidumbre de migrar, así como el costo de llegar (no necesariamente el de "pasar"), que incluso puede ser financiado por un familiar del otro lado. 
En el polo opuesto estaba la gran mayoría de los hogares encuestados (73\%), que afirmaron que su situación en educación se había mantenido sin cambio. Mientras que el 20\% restante reportó una mejoría en este aspecto. Seguramente, en este segmento tenemos a los hogares que tenían a la educación como un valor importante dado que la percepción está influenciada por la cultura y valores de las personas. Un ejemplo, son los tres casos en que el padre migró para solventar los gastos de educación de los hijos, porque en su visión de futuro sus hijos deberían ir a la escuela para no tener que migrar.

En cuanto a la vivienda, la gran mayoría (84\%) consideró que las remesas habían contribuido al mejoramiento de sus condiciones, esto tenía cierta relación con los datos obtenidos del gasto en construcción. Aunque también hubo una percepción del 16\% de los encuestados, en el sentido de que su vivienda estaba igual que antes de la migración, esto pudo relacionarse con el hecho de que el migrante tenía poco tiempo de haberse ido y casi la totalidad de las remesas se destinaban al consumo del hogar.

El primer sentimiento que genera la creación de un patrimonio es el de seguridad en el futuro, principalmente en el aspecto financiero. Por lo tanto, la seguridad a la que se hace referencia en esta pregunta es la de tipo financiero. Esta consiste en que el hogar tiene la certidumbre de que podrá hacer frente a alguna contingencia futura y que cuando llegue la etapa de la vejez tendrá suficiente ingreso para mantener su consumo, es decir, no necesitará que otros miembros del hogar más jóvenes lo mantengan. Dentro de los hogares con migrantes esta racionalidad tiene sus matices, por la idiosincrasia familiar se esperaría que los jóvenes "vieran" por los viejos, pero los jóvenes que están trabajando en los Estados Unidos, también están aprendiendo parte de la cultura del retiro laboral en aquel país y ya no esperarían todos que sus hijos los "vieran" en la vejez, aunque ellos sí a sus padres. Esto estaría incentivando la formación de patrimonio en los hogares con migrantes y por ende generando seguridad sobre el futuro. Al respecto los hogares encuestados contestaron lo siguiente, el 53\% afirmó que las remesas habían mejorado la seguridad futura del hogar, mientras que el $47 \%$ restante tuvo una percepción más conservadora y modesta de su situación y declaró que las remesas habían mantenido igual la seguridad que tenían sobre su futuro. Es posible que esto también se relacione con la antigüedad en la migración dentro del hogar y la edad promedio de sus miembros, además del número de miembros del hogar y de las expectativas a futuro de cada uno de ellos con relación a migrar de forma temporal o permanente. Esto puede ser objeto de un estudio y análisis más detalla- 
do para identificar oportunidades de políticas públicas que contribuyan a mejorar el bienestar de las familias con migrantes.

\section{Gráfica 3. Efectos de las remesas en diversos rubros}

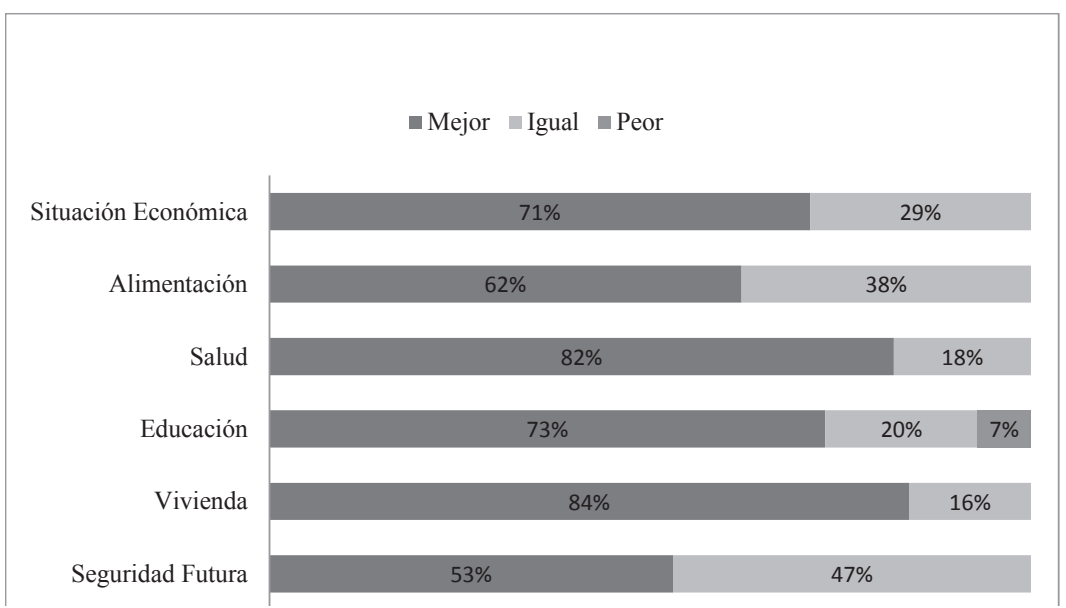

Fuente: Elaboración propia con base en cuestionarios para la MCS. San Jerónimo Coyula, 2006

\section{Relación entre monto de las remesas, antigüedad en la migración y percepciones de bienestar}

En esta última parte se realizó un ejercicio para encontrar la vinculación entre los niveles de las remesas con las percepciones sobre la situación económica, alimentación, salud, educación, vivienda, formación de patrimonio y seguridad en el futuro, mediante el uso de tablas de contingencia, se utilizó la prueba de chi cuadrada para probar la hipótesis de no relación entre las variables con un nivel de confianza del $95 \%$. Para realizar el cruce cumpliendo con las condiciones de frecuencias esperadas por casillas, se clasificaron los montos de remesas en dos grupos: bajos y altos ingresos, los rangos se establecieron teniendo como referencia la media del conjunto estudiado. Los resultados de la prueba chi cuadrada de independencia se resumen en el siguiente cuadro. 
Cuadro 1. Resultados de tablas de contingencia

\begin{tabular}{|c|c|}
\hline Monto de remesas con: & Sig Chi cuadrada \\
\hline Situación económica & 0.497 \\
\hline Alimentación & 0.848 \\
\hline Salud & 0.210 \\
\hline Educación & 0.129 \\
\hline Vivienda & 0.295 \\
\hline Seguridad en el futuro & 0.731 \\
\hline Antigüedad en la migración con: & 1.000 \\
\hline Seguridad en el futuro & 0.011 \\
\hline Situación económica & Coyul 2006 \\
\hline
\end{tabular}

Fuente: Cálculos propios basados en cuestionarios para la MCS. San Jerónimo Coyula. 2006

Cruzando las remesas con las percepciones sobre sus efectos en la situación económica de los hogares, se observó que tanto en el grupo de bajos como de altos ingresos por remesas, la mayoría percibió que mejoró su situación económica al recibirlas, lo cual se comprobó con el resultado de la prueba chi cuadrada, al no rechazarse la hipótesis de independencia entre las variables.

Al cruzar las remesas con las percepciones sobre la alimentación, se observó que la mayoría de los hogares percibieron mejoría, pero ella no dependió del monto de las remesas recibidas, los resultados de la prueba chi cuadrada demostraron que para la mayoría de los grupos, tanto de bajos como de altos ingresos por remesas, había mejorado su alimentación con su llegada.

En el tema de salubridad, cruzando las remesas con las percepciones sobre la salud, se observó que la mayoría de los hogares de altos y bajos ingresos por remesas sintieron mejoría. Sin embargo, la prueba chi cuadrada mostró que la mejoría no dependió del monto recibido.

Cruzando las remesas con las percepciones sobre la educación se observó algo distinto en el efecto de las remesas, pero similar en cuanto a la independencia de las variables de acuerdo con la prueba estadística, para la mayoría de los hogares sin importar la cantidad que recibían de remesas, los efectos fueron poco notorios, incluso en el nivel bajo de ingreso el efecto en educación se percibió como negativo en algunos hogares. Esto tiene una explicación probable porque las remesas han llevado a que los adolescentes quieran irse a Estados Unidos al terminar la secundaria y en la óptica de la mayoría de los hogares invertir en educación más allá de la básica, no favorecía para migrar y menos como un medio para movilizarse socialmente en México. Los hogares que percibieron mejoría en sus condiciones de educación fueron principalmente del grupo de ingresos bajos.

El tema de casa arroja la siguiente información: cruzando las remesas con las percepciones sobre las condiciones de vivienda, se observó que en 
todos los niveles de ingreso las remesas habían mejorado en la mayoría de los hogares, lo cual se comprobó con la prueba estadística que mostró la independencia de las dos variables. Los rezagos en este rubro estaban ubicados principalmente en el nivel bajo de ingreso, donde todavía no eran suficientes los recursos que llegaban para ampliar, remodelar o construir la casa.

Cruzando las remesas con las percepciones sobre la seguridad en el futuro se observó que en ambos grupos de ingreso la mayoría de los hogares se sentía con más seguridad frente a la incertidumbre en el futuro, lo cual también se comprobó con la prueba estadística de independencia. No obstante hay que resaltar que el número de hogares que percibieron mejoría y el que consideró que se mantuvo igual, no fue tan diferente como en los demás rubros del bienestar.

De lo anterior se puede concluir que la percepción de mejoría en los rubros de alimentación, salud, educación, vivienda, situación económica y seguridad en el futuro, si bien es influida por las remesas en mayor o menor medida, de acuerdo con los resultados de la prueba estadística no hay suficiente evidencia para afirmar que depende del monto de las mismas, al menos en este caso de estudio. En realidad difícilmente se puede decir más al respecto, es necesario también incorporar en el análisis la variable antigüedad de la migración, debido a la certidumbre que puede dar en términos de regularidad en el envío de las remesas (por empleo e ingreso más estable en el lugar de destino) y superación de la etapa de sobrevivencia de la familia, para esto se cruzó la antigüedad en la migración dividida en dos grupos, los que tenían hasta 5 años y los que tenían 6 años y más de haber iniciado su experiencia migratoria. Los resultados de este cruce arrojaron que aunque la antigüedad de la migración parecía más notoria en la percepción de mejoría de los hogares sobre la seguridad en el futuro, los resultados de la prueba estadística mostraron una perfecta independencia, por lo tanto, la antigüedad en la migración no era significativa para que los hogares percibieran mayor seguridad en el futuro. Finalmente y a diferencia de lo anterior, el efecto de la antigüedad en la migración sí influía de manera contundente en la percepción sobre la situación económica del hogar. Entre más años se tenía de experiencia migratoria, el hogar percibía que su situación económica había mejorado significativamente, esta fue la única prueba estadística donde se pudo rechazar la hipótesis de independencia entre las variables, a un nivel de confianza del 95\%. 


\section{Conclusiones}

Para los hogares, las remesas son medios para sobrevivir en un primer momento y para formar un patrimonio más adelante, generalmente son un complemento de los ingresos habituales del hogar y en muchos casos llegan a ser los más importantes. Como en cualquier hogar, la necesidad de mejorar su bienestar ha sido el principal motivo para decidir sobre la migración. La falta de empleo, pero sobre todo bien remunerado, aunado a percepciones de pobreza relativa en la comunidad, han provocado que gran parte de los jóvenes emigren, situación que se cataloga y se torna compleja cuando existe una red social formada con el tiempo, que disminuye los costos de la migración y magnifica los beneficios. Este parece ser el caso de muchas de las poblaciones en México, que a partir de las crisis en los años ochenta vieron en la migración una forma de sobrevivir y progresar. La región de Atlixco en Puebla, incorporada a esta dinámica tiene en la comunidad de San Jerónimo Coyula un importante ejemplo de migración hacia los Estados Unidos, donde una buena parte de los hogares recibe y usa remesas. En este trabajo se ha reportado el uso de las remesas y su relación con el desarrollo de los hogares, entendido como el mejoramiento de las condiciones de vida.

A partir del análisis realizado fue posible concluir que los hogares gastaban sus remesas respondiendo a las necesidades que motivaron la migración, si la remesa era parte de un salario internacional, entonces su gasto se hacía primero en lo básico y después en lo demás, pudiendo llegar a ahorrar y a invertir en capital humano, salud y educación, en usos patrimoniales como la remodelación o construcción de la casa, la compra de tierras y otros activos físicos, lo cual afectaba la percepción del hogar sobre su bienestar en términos económicos, de salud, educación, vivienda y seguridad en el futuro. Sin embargo, los efectos de las remesas sobre la percepción que tenían los hogares en la mejoría de su bienestar no fueron uniformes. Desglosando por aspectos resalta que un estudio como éste lo que reporta son generalidades, pero se tiene que reconocer que cada hogar tiene sus objetivos y sus formas de administrar las remesas, dependiendo de la etapa de desarrollo o del ciclo que esté viviendo. Con este acotamiento se puede afirmar que en el caso de los hogares encuestados, las remesas mejoraron la situación económica de los hogares beneficiados en la mayoría de los casos, y cuando no fue así reconocieron que continuó igual. En los aspectos de alimentación y salud sucedió algo parecido, aunque la mejoría fue más notoria en los hogares que recibían menores cantidades de 
remesas. Con respecto a la educación el resultado fue contrastante, para la mayoría de los hogares las remesas no mejoraron su bienestar en términos de educación, es más en los hogares con menores ingresos algunos opinaron que lo había empeorado, muy probablemente por el aspecto negativo en los muy jóvenes de "irse" lo más pronto a trabajar del otro lado. En el mismo sentido, se puede afirmar que la mayoría de los hogares utilizaban muy poco las remesas para educación, lo que puede llevar a plantear que al menos en esta comunidad la migración no perseguía en la mayoría de los casos mejorar las condiciones de educación de los miembros de los hogares.

En cuanto a los efectos de las remesas sobre las condiciones de vivienda, la mejoría fue notoria en la mayoría de los hogares, en aquellos donde las condiciones permanecían igual, era porque recibían menores ingresos y los recursos no habían llegado a ser lo suficientes. El otro aspecto fundamental para la tranquilidad de los hogares era la seguridad que pudieran sentir sobre su futuro. Al respecto la mayoría de los hogares opinó que había mejorado, siendo más notorio en los hogares que recibían más remesas. Lo cual resultó lógico porque finalmente si se recibía un mayor flujo de dinero era posible expandir la frontera de consumo, ahorro y formación de patrimonio.

En el mismo sentido resultaron las pruebas estadísticas, que evidenciaron que sin importar el monto de las remesas las percepciones de mejoría en el bienestar de la mayoría de los hogares eran notorias, sólo la antigüedad de la migración influía significativamente en la percepción de la situación económica de los hogares. Lo anterior comprueba el gran efecto que tiene el aumento del ingreso del hogar al recibir remesas.

Al menos para esta comunidad, pero sin olvidar que hay muchas parecidas a ésta en la realidad del fenómeno migratorio en México, es posible concluir que los hogares administraban sus remesas de manera racional, cubriendo primero sus necesidades básicas y después las secundarias, siguiendo de manera parecida el planteamiento de A. Maslow. En esta perspectiva es fundamental que las familias cuenten con una mínima cultura financiera para que puedan aprovechar mejor sus remesas.

Con los resultados de este estudio resulta evidente que para fines de política pública, es importante dejar muy claro que las remesas son recursos privados que llegan a las familias y que lo más común es que las gasten como cualquiera de los asalariados que trabajan en el país, con la diferencia de que efectivamente por la cantidad y frecuencia en que las reciben, muy pronto se convierten en un flujo para los hogares que les permite superar con cierta rapidez estados de carencia básica, pero que no por ello se debería pensar que los hogares migrantes deberían de convertirse necesariamen- 
te en empresarios o algo parecido para salir de la pobreza en México. Las condiciones en que ahorran, pero sobre todo sus expectativas para formar un patrimonio que les dé seguridad en el futuro, pasan por invertir en activos productivos, pero no precisamente en empresas. Si se observa en qué se gastan las remesas, queda claro que son parte de un salario internacional y que por lo mismo los que deciden sobre su uso son los migrantes y sus familias, que deben tener un trato de agente económico capaz no sólo de consumir, sino de formar un patrimonio, de ser sujetos de crédito y de tener tratos justos en sus operaciones comerciales. Ser susceptibles de incentivos y condiciones para canalizar su ahorro hacia fines empresariales. ${ }^{7}$

La política pública debería crear condiciones de confianza y transparencia, para incentivar un comportamiento más comunitario y de participación de los ciudadanos en las decisiones de las comunidades para hacer más economía local, esto debe estar enmarcado en una política de desarrollo regional que fortalezca la infraestructura y procesos de integración productiva, para incentivar a su vez la participación económica de los hogares, pero sobre todo de los migrantes que están del otro lado y generalmente deciden sobre el uso de las remesas.

En síntesis, las remesas vistas en los hogares encuestados de San Jerónimo Coyula tuvieron un efecto positivo en su desarrollo y bienestar, por lo que se infiere que la migración ha sido y seguirá siendo la estrategia de los hogares para integrarse a los flujos de la globalización y alejarse de los riesgos del empobrecimiento en el lugar de origen. Pero sería mejor si contaran con el apoyo de una política de desarrollo regional, que les diera la oportunidad de integrar sustancialmente las remesas en una estrategia de crecimiento de la economía local, en la que su consumo y/o su inversión sirvieran para generar empleo en sus propias comunidades.

7 Para que las remesas se inviertan en usos productivos se requiere de un estado que garantice los derechos de propiedad, estabilidad política y seguridad para la inversión, condiciones estructurales y macroeconómicas favorables para el desarrollo del mercado local, oportunidades para inversiones rentables y condiciones personales como experiencia en el manejo de negocios, como principales factores (Russell, 1992). 


\section{Bibliografía}

Ahlburg, Dennis A., 1991, Remittances and their lmpact: A Study of Tonga and Western Samoa, Canberra, Australia, National Center for Development Studies.

Borjas, George, 2000, "Economics of migration", Internacional Enciclopedia of the Social and Behavioral Sciences, Section No. 3.4, Article No. 38.

Canales, Alejandro, 2008, Vivir del norte. Remesas, Desarrollo y pobreza en México, México: SEGOB-CONAPO.

Canales, Alejandro, 2004, “Las remesas de los migrantes”, en Germán Zárate Hoyos (coord.), Remesas de los mexicanos y centroamericanos en Estados Unidos, Problemas y Perspectivas, México, El Colegio de la Frontera Norte.

Corona Jiménez, M.A., 2009, “Household Development in Tlapanalá. A comparative study between households receiving remittances and households not receiving remittances", Journal of Poverty. Innovations on Social Political \& Economic Inequalities, vol. 13, pp. 331-349.

Corona Jiménez, M.A., 2008, “La economía de los Migrantes Poblanos en Nueva York", Migraciones, vol., 24, pp. 57-89.

Corona Jiménez, M.A., 2007, “La economía de Tlapanalá”, Migraciones Internacionales, vol. 4, pp. 93-120.

Cortina, Regina y Mónica Gendreau, 2004, Poblanos en Nueva York. Migración rural, educación y bienestar. México, Universidad Iberoamericana Puebla.

Durand, Jorge y Douglas Massey, 2003, Clandestinos. Migración México-Estados Unidos en los albores del siglo XXI, México, Miguel Ángel Porrua-Universidad Autónoma de Zacatecas.

Ferrer-i-Carbonell, Ada, 2002a, "Income and Well-being” Tinbergen Institute Discussion Papers, núm. 02-019/3, Holanda, Tinbergen Institute.

Ferrer-i-Carbonell, Ada, 2002b, "Subjective Questions to Measure Welfare and Wellbeing" Tinbergen Institute Discussion Papers, núm. 02-020/3, Holanda, Tinbergen Institute. 
Lozano Ascencio, Fernando, 2004, “Tendencias Recientes de las Remesas de los Migrantes Mexicanos en Estados Unidos", Working Paper 99, San Diego, University of California.

Maslow, Abraham H., 1943, "A theory of Human Motivation”, Psychological Review, vol. 50, pp. 370-396.

Ochoa León, Sara María, 2004, “Remesas: Un acercamiento a sus impactos sobre la pobreza y el desarrollo", Boletín No. 7, México, Centro de Estudios Sociales y de Opinión Pública.

Orozco, Manuel, 2004, Remittances to Latin America and the Caribbean: issues and perspectives on development, Washington, D.C., OEA.

Ratha, Dilip, 2003, “Worker's Remittances: An important and stable source of External Development Finance". Global Development Finance: Striving for Stability in Development Finance, Washington D.C., World Bank, pp. 157-175.

Ravallion, Martin y Michael, Lokshin, 2000, "Identifying welfare effects from subjective

Questions", World Bank Policy Research Working Papers. Washington, D.C., núm. 2301.

Russell, Sharon S., 1992, "Migrant Remittances and Development", InternationaI Migration, vol. xxx, pp. 3-4.

Seligman, M.E.P., 1999, “The Presidents Address. APA.1998”, American Psychologist, vol. 54, pp. 559-562.

Stark, Oded y E. Taylor, 1991, “Migration Incentives, Migration Types: The Role of Relative Deprivation”, Economic Journal, vol. 101, pp. 1163-1178.

Taylor, J.E., 1999, “The New Economics of Labour Migration and the Role of Remittances in the Development Process", International Migration, vol. 37, pp. 6388.

Taylor, J.E., 1992, “Remittances and Inequality Reconsidered: Direct, Indirect and Intertemporal Effects", Journal of Policy Modeling, vol. 14, pp. 187-208.

Recibido el 8 de agosto de 2012 Aceptado el 10 de julio de 2013 